11

\title{
Тепловое излучение графена
}

\author{
() С.Ш. Рехвиашвили ${ }^{1}$, W. Strek $^{2}$ \\ ${ }^{1}$ Институт прикладной математики и автоматизации КБНЦ РАН, \\ 360002 Нальчик, Россия \\ ${ }^{2}$ Institute of Low Temperature and Structure Research, Polish Academy of Sciences, \\ 50-422 Wrocław, Poland \\ e-mail: rsergo@mail.ru,w.strek@intibs.pl
}

Поступила в редакцию 12.05.2021 г.

В окончательной редакции 05.07.2021 г.

Принята к публикации 05.07.2021 г.

Рассмотрена задача о тепловом излучении идеального графена. В качестве основы взяты теоретические представления о поверхностных электромагнитных волнах и поверхностном импедансе. Вычислена интенсивность теплового излучения графена как функция частоты внешнего излучения. Обсуждается роль размерного эффекта теплового излучения в формировании широкополосного („белого“) излучения графеновой пены.

Ключевые слова: графен, тепловое излучение, поверхностные электромагнитные волны, коэффициенты Френеля, поляризация, поверхностный импеданс.

DOI: 10.21883/OS.2021.10.51497.2284-21

\section{Введение}

Теория теплового излучения графена в окончательном виде пока не построена. Препятствием являются двумерность этого материала и сложности в применении классической электродинамики сплошной среды. Отметим, что аналогичные проблемы возникают в теории дисперсионных сил Ван-дер-Ваальса и запаздывающих сил Казимира для графена и углеродных нанортубок [1-3].

Графен как абсолютно черное тело рассматривался в $[4,5]$. Метод основывался на расчетах плотности фотонных состояний в зависимости от размерности евклидова пространства [6,7]. Полученные в [4] результаты нельзя считать в полной мере удовлетворительными. Так, авторы не описали механизм теплового излучения в двумерном случае. Как следствие, не был рассмотрен процесс испускания фотонов, и поэтому плотность мощности черного излучения осталась невычисленной. Этот пробел устранен в [5]. Был выведен аналог закона Стефана-Больцмана для двумерного черного тела. В качестве такого тела можно взять кольцо из тонкой сверхпроводящей проволоки [8]. Фотоны, отражаясь от проволоки, будут заполнять пространство внутри кольца. Такая модель применима к идеальному графену по следующим причинам. При нормальном падении электромагнитных волн графен является почти прозрачным, т.к. пропускает $97.7 \%$ излучения в широком частотном диапазоне видимого спектра [9-11]. Почти вся остальная часть излучения отражается. Тоже самое относится и к инфракрасном диапазону, поскольку здесь выполняется условие $\lambda / d \gg 1$, где $\lambda-$ длина волны излучения, $d=0.335 \mathrm{~nm}$ эффективная толщина графена. В этой связи из закона Кирхгофа следует, что тепловое излучение с поверхности графена практически отсутствует. В пользу данного заключения косвенно свидетельствуют антифрикционные взаимодействия [12] и малые значения дисперсионных сил [13] в контактах, образованных плоскими листами графена. Ситуация совершенно иная, если внешнее излучение попадает на торец графена. В этом случае можно вести речь о поверхностных электромагнитных волнах (в общем случае с произвольной поляризацией), которые распространяются в плоскости графена. Для этих волн характерны скинэффект, поглощение и тепловое излучение.

В $[14,15]$ изучалось широкополосное („белое“ ${ }^{6}$ излучение графеновой пены. Образцы подвергались воздействию сфокусированного инфракрасного излучения. Было выявлено, что область свечения ограничивается размерами фокального пятна. С увеличением плотности мощности внешнего излучения интенсивность вторичного излучения образцов сначала экспоненциально возрастает, а затем достигает насыщения. Укажем, что математически такая зависимость описывается логистической функцией [16]. При высоких значениях интенсивности внешнего излучения имеются широкие полосы как видимого, так и инфракрасного излучения. Согласно [5], излучение имеет тепловую природу и происходит преимущественно от торцов стенок графеновой пены. Характер теплового излучения условной твердой пены в определенной мере передает модельный эксперимент, результаты которого представлены на рис. 1. В качестве образца был взят скомканный лист черной копировальной бумаги (рис. 1,a). Образец равномерно нагревался потоком горячего воздуха. Нагрев и остывание регистрировались инфракрасной камерой (рис. 1, it b). Этот простой эксперимент показывает, что в первую очередь остывают внешние выступы и изломы. Внутри образца сохраняются горячие области, которые со временем также остывают. В реальных образцах пены этот про- 

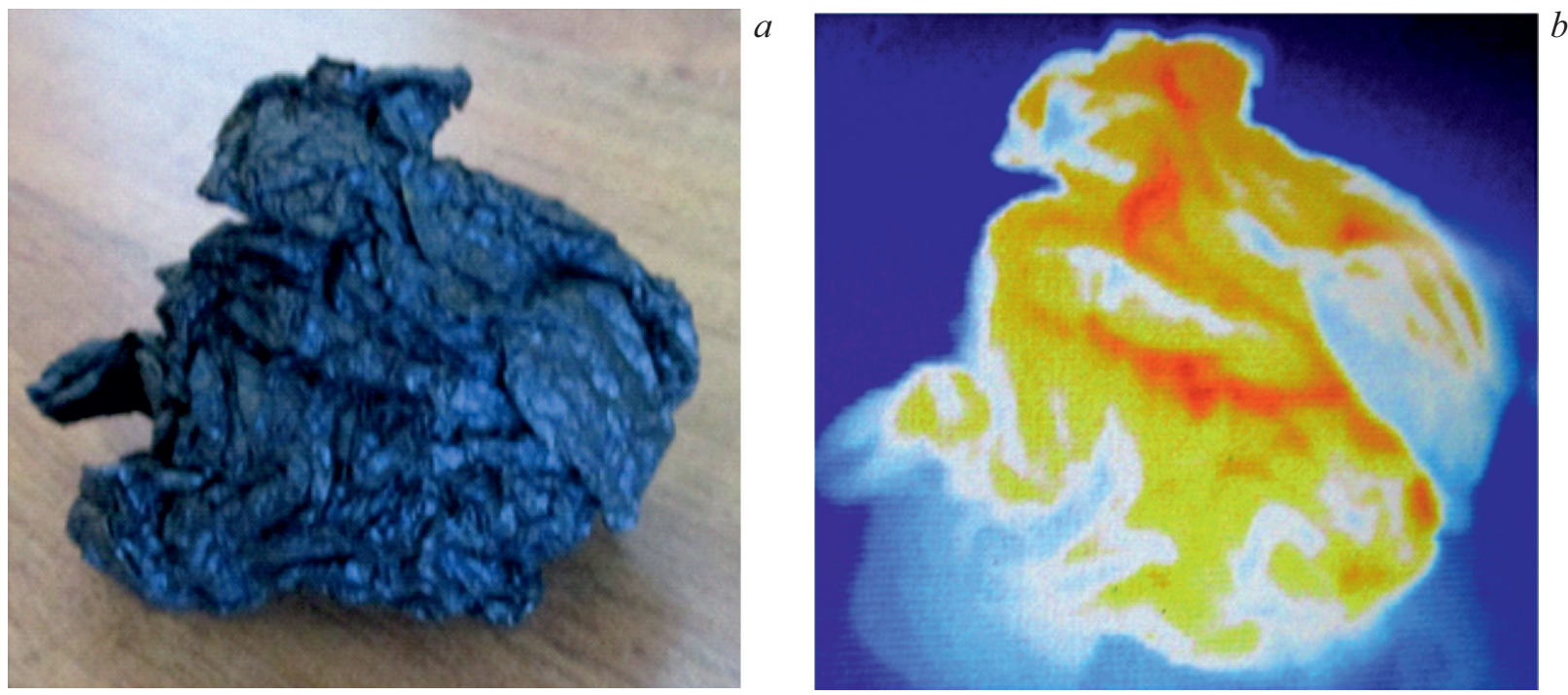

Рис. 1. Модельный эксперимент по тепловому излучению графеновой пены.

цесс, очевидно, происходит значительно быстрее из-за высокой теплопроводности графена.

В настоящей работе развиваются идеи $[5,8]$ и рассчитывается интенсивность теплового излучения идеального графена с учетом его физических свойств и характеристик внешнего излучения. За основу принято представление об электромагнитных волнах, которые распространяются вдоль поверхности и испускаются в окружающее пространство с краев графена.

\section{Теоретическая модель}

Рассмотрим излучение с частотой $\omega$, которое падает из вакуума на торец листа графена. Плоскость падения совпадает с плоскостью графена. Различаются два случая: 1) вектор напряженности электрического поля перпендикулярен плоскости графена ( $s$-поляризация); 2) вектор напряженности электрического поля лежит в плоскости графена ( $p$-поляризация). Для этих двух случаев коэффициенты отражения равны [17]:

$$
\begin{gathered}
R_{\perp}=\left|\frac{\cos \theta-\sqrt{\varepsilon_{\perp}-\sin ^{2} \theta}}{\cos \theta+\sqrt{\varepsilon_{\perp}-\sin ^{2} \theta}}\right|^{2}, \\
R_{\|}=\left|\frac{\varepsilon_{\|} \cos \theta-\sqrt{\varepsilon_{\|}-\sin ^{2} \theta}}{\varepsilon_{\|} \cos \theta+\sqrt{\varepsilon_{\|}-\sin ^{2} \theta}}\right|^{2},
\end{gathered}
$$

где $\theta-$ угол падения относительно нормали, $\varepsilon_{\|}$и $\varepsilon_{\perp}$ диэлектрические проницаемости для двух направлений поляризации. Коэффициент отражения неполяризованного излучения

$$
R=\frac{1}{2}\left(R_{\perp}+R_{\|}\right) .
$$

По закону Кирхгофа интенсивность теплового излучения связана с интенсивностью излучения от абсолютно черного тела соотношением

$$
d I=I_{0} p(1-R) d \Omega
$$

где $I_{0}$ - интенсивность излучения абсолютно черного тела, вычисленная в [5], p - параметр, учитывающий изотропию излучения, $d \Omega$ - элемент телесного угла. В нашем случае излучение происходит в полуокружность, поэтому для двумерного телесного угла будем иметь $d \Omega=d \theta$. С учетом этого для интенсивности излучения получаем

$$
I=I_{0} p \int_{0}^{\pi / 2}[1-R(\theta)] d \theta=I_{0} p\left[\frac{\pi}{2}-\int_{0}^{\pi / 2} R(\theta) d \theta\right],
$$

где функция $R(\theta)$ вычисляется из (1)-(3).

Перейдем к оценке интеграла в (5). Сначала обсудим вклад волн с s-поляризацией. Электропроводность, обусловленная подвижными электронами, существует только вдоль поверхности графена, поэтому для этих волн графен подобен неполярному диэлектрику. Если предположить, что для данного направления поляризации $\varepsilon_{\perp} \rightarrow 1$, то из $(1)$ получается $R_{\perp} \rightarrow 0$. Данное предположение качественно представляется правильным если вектор напряженности электрического поля перпендикулярен плоскости графена, то диссипация поля будет происходить лишь за счет поляризации смещения, т.е. скорее всего будет мала. Следовательно, волны с $s$-поляризацией будут распространяться по поверхности графена на большие расстояния и слабо участвовать в нагреве. В случае волн с $p$-поляризацией возникает определенная аналогия с металлами, поэтому физическая картина выглядит по-другому. Учитывая высокое значение электропроводности графена, формула (2) может быть упрощена с применением приближения 
Леонтовича. Оно заключается в том, что распространение электромагнитного поля вне хорошо проводящего тела при однородных граничных условиях можно с определенной точностью свести к решению уравнений Максвелла для внешнего пространства [17]. Другими словами, электромагнитное поле внутри тела можно не рассматривать. Приближение считается справедливым при таких условиях: а) проникновение в тело и длина волны малы по сравнению с длиной волны в окружающем пространстве, по сравнению с расстоянием от источника внешнего поля и по сравнению с радиусом кривизны поверхности; б) изменения диэлектрической и магнитной проницаемостей на расстоянии проникновения волны малы.

Введем функцию

$$
Z=\frac{Z_{0}}{\sqrt{\varepsilon_{\|}}}=Z_{0} \xi,
$$

где $Z_{0} \approx 120 \pi \approx 377 \Omega$ - волновое сопротивление вакуума, $\mathcal{\zeta}$ - поверхностный импеданс графена. Импедансное граничное условие имеет вид

$$
\mathbf{H}=\frac{1}{Z}[\mathbf{n}, \mathbf{E}],
$$

где $\mathbf{E}$ и $\mathbf{H}-$ компоненты электрического и магнитного полей снаружи графена, $\mathbf{n}$ - единичный вектор нормали, направленный от границы вглубь материала. Из (6) и (7) получается формула для коэффициента отражения волны от торца графена

$$
R_{\|}=\left|\frac{\cos \theta-\xi}{\cos \theta+\xi}\right|^{2} .
$$

В (8) необходимо определить $\xi$ в зависимости от действительной $\varepsilon_{\|}^{\prime}$ и мнимой $\varepsilon_{\|}^{\prime \prime}$ частей диэлектрической функции $\varepsilon_{\|}$. Общее выражение имеет вид

$$
\begin{gathered}
\xi=\xi^{\prime}+i \xi^{\prime \prime}=\frac{\cos \left(0.5 \operatorname{arctg}\left(\varepsilon_{\|}^{\prime \prime} / \varepsilon_{\|}^{\prime}\right)\right)}{\sqrt[4]{\varepsilon_{\|}^{\prime 2}+\varepsilon_{\|}^{\prime \prime 2}}} \\
-i \frac{\sin \left(0.5 \operatorname{arctg}\left(\varepsilon_{\|}^{\prime \prime} / \varepsilon_{\|}^{\prime}\right)\right)}{\sqrt[4]{\varepsilon_{\|}^{\prime 2}+\varepsilon_{\|}^{\prime \prime 2}}} .
\end{gathered}
$$

Как и требуется, в (9) имеют место условия $\xi^{\prime}>0$ и $\xi^{\prime \prime}<0$. На низких частотах должно выполняться условие $\varepsilon_{\|}^{\prime \prime} \gg \varepsilon_{\|}^{\prime}$, поэтому из (9) находим

$$
\xi \approx(1-i) \frac{1}{\sqrt{2 \varepsilon_{\|}^{\prime \prime}}} .
$$

Далее воспользуемся моделью оптической проводимости графена [18], в соответствии с которой диэлектрическая проницаемость есть

$$
\varepsilon_{\|}=\varepsilon_{\|}^{\prime}+i \varepsilon_{\|}^{\prime \prime}=1+i \frac{\pi c \alpha}{\omega d},
$$

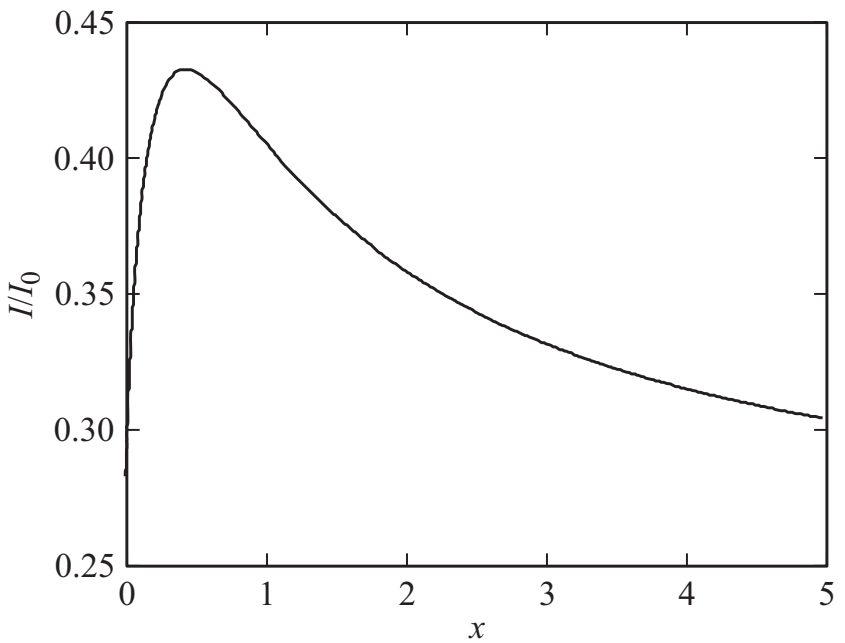

Рис. 2. Относительная интенсивность излучения графена, рассчитанная по формуле (14).

где $\alpha-$ постоянная тонкой структуры, $c-$ скорость света в вакууме. При записи (11) подразумевалось, что оптическая проводимость в ближней инфракрасной области и при ненулевой температуре имеет универсальное значение [18]: $\sigma=q^{2} /(4 \hbar d) \approx 1.82 \cdot 10^{5} \mathrm{~S} / \mathrm{m}$, где $q$ - заряд электрона, $\hbar-$ постоянная Планка. Из (10) и (11) получаем

$$
\begin{gathered}
\xi=(1-i) \sqrt{\frac{\omega d}{2 \pi c \alpha}}, \\
\omega \ll \frac{\pi c \alpha}{d} .
\end{gathered}
$$

Условие (13) надежно выполняется для всего инфракрасного диапазона электромагнитных волн $(\omega /(2 \pi) \ll 3265 \mathrm{THz})$.

Если излучение происходит с границы хорошо проводящего двумерного полупространства в вакуум, то в (4) и (5) нужно принять $p=1 / \pi$. Из (5), (8) и (12) окончательно приходим к формуле

$$
\begin{gathered}
I=I_{0}\left[\frac{1}{4}+\frac{2 x}{\pi} \int_{0}^{\pi / 2} \frac{\cos (\theta) d \theta}{(\cos (\theta)+x)^{2}+x^{2}}\right], \\
x=\sqrt{\frac{\omega d}{2 \pi c \alpha}} .
\end{gathered}
$$

Интеграл в (14) существует, однако не выражается через элементарные функции. При больших $x$ он имеет асимптотику $1 / x$. Результаты расчета по (14) показаны на рис. 2. У функции имеется один максимум, которому отвечают значения $x=0.441$ и $I / I_{0}=0.433$. В $[14,15]$ использовался инфракрасный лазер с длиной волны $975 \mathrm{~nm}$. Для этого случая $x=0.217$ и $I / I_{0}=0.414$, т.е. относительная интенсивность излучения незначительно отличается от найденного максимума. Отсюда 


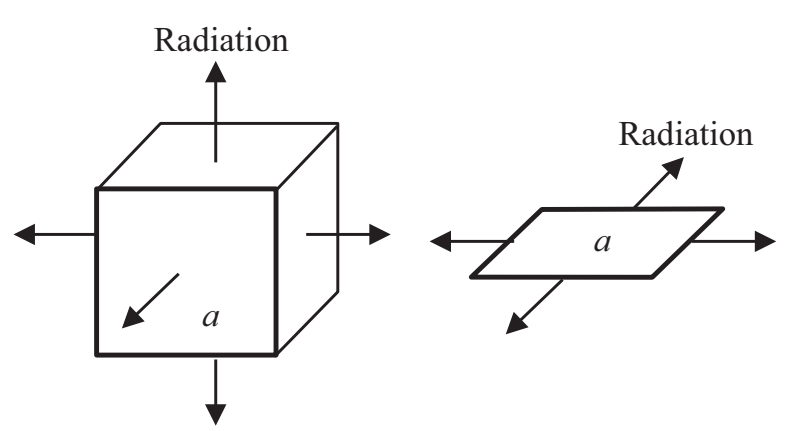

Рис. 3. Излучающие микрочастицы.

можно заключить, что выбранный в $[14,15]$ лазер теоретически хорошо подходит для нагрева графеновой пены. В точке максимума частота излучения и толщина скин-слоя, определяемая по формуле $\delta=\sqrt{2 c d /(\pi \alpha \omega)}$, составляют $1270 \mathrm{THz}$ и $33 \mathrm{~nm}$, что удовлетворяет всем необходимым условиям применимости теории. При определенных значениях температуры и частоты внешнего излучения малое значение $\delta$ может указывать на аномальный характер скин-эффекта [19]. Приближение Леонтовича в данном случае работает, но формула (12) для поверхностного импеданса должна быть дополнительно обоснована или даже пересмотрена.

Наконец, рассмотрим размерный эффект черного излучения на конкретном примере. Запишем законы Стефана-Больцмана для трехмерного и двухмерного (см. $[5,7])$ случаев

$$
P_{3 \mathrm{D}}=\frac{\pi^{2}\left(k_{\mathrm{B}} T\right)^{4}}{60 c^{2} \hbar^{3}} S, \quad P_{2 \mathrm{D}}=\frac{2 \xi(3)\left(k_{\mathrm{B}} T\right)^{3}}{c(\pi \hbar)^{2}} L,
$$

где $k_{\mathrm{B}}$ - постоянная Больцмана, $T$ - температура, $S-$ площадь, $L-$ периметр,

$$
\zeta(3)=\sum_{n=1}^{\infty} n^{-3} \approx 1.202
$$

- постоянная Апери. Пусть при температуре $T=1000 \mathrm{~K}$ имеются две микрочастицы со свойствами абсолютно черного тела в форме куба и квадрата (рис. 3), для которых $S=6 a^{2}$ и $L=4 a$, где $a=1 \mu \mathrm{m}-$ линейный размер микрочастиц. Расчеты дают значения $P_{3 \mathrm{D}}=340 \mathrm{nW}$ и $P_{2 \mathrm{D}}=769 \mathrm{nW}$. Видно, что мощности излучения одинаковых по размеру частиц отличаются более, чем в два раза. С уменьшением температуры и размеров частиц этот эффект проявляется еще сильнее. Таким образом, имеются основания полагать, что размерный эффект теплового излучения может являться причиной низкотемпературного и яркого свечения графеновой пены, которое наблюдалось экспериментально в $[14,15]$.

\section{Заключение}

В настоящей работе с применением понятия поверхностных электромагнитных волн разработана качественно новая теоретическая модель теплового излучения графена с учетом его электропроводности и частоты внешнего инфракрасного излучения. Предполагалось, что основной вклад в радиационный нагрев вносят электромагнитные волны с $p$-поляризацией, у которых вектор напряженности электрического поля лежит в плоскости графена. Для этих волн использовалось импедансное граничное условие, что оправдывается высокой электропроводностью и малой толщиной скин-слоя.

По данной работе с учетом [5,8] можно сформулировать следующие выводы. Широкополосное излучение графеновой пены, обнаруженное экспериментально в $[14,15]$, вероятнее всего вызвано не сильным повышением температуры, а физическими свойствами материала и проявлением размерного эффекта теплового излучения. Не исключается также температурноактивированная люминесценция графеновой пены. Без учета вклада волн c s-поляризацией интенсивность теплового излучения идеального графена составляет свыше $43 \%$ от интенсивности излучения двумерного абсолютно черного тела. Улучшенная теоретическая модель теплового излучения графена должна учитывать электромагнитные волны с $s$-поляризацией и температурные зависимости характеристик материала. По нашему мнению, имеются перспективы применения широкополосного излучения графеновой пены в оптоэлектронике и плазменной электронике на основе графена, которые сейчас активно развиваются [20].

\section{Конфликт интересов}

Авторы заявляют, что у них нет конфликта интересов.

\section{Список литературы}

[1] Bordag M., Klimchitskaya G.L., Mohideen U., Mostepanenko V.M. Advances in the Casimir Effect. Oxford University Press, 2009. 749 p.

[2] Gomez-Santos G. // Phys. Rev. B. 2009. V. 80. P. 245424. doi 10.1103/PhysRevB.80.245424

[3] Drosdoff D., Woods L.M. // Phys. Rev. B. 2010. V. 82. P. 155459. doi 10.1103/PhysRevB.82.155459

[4] Heetae Kim, Seong Chu Lim, Young Hee Lee // Physics Letters A. 2011. V. 375. N 27. P.2661-2664. doi 10.1016/j.physleta.2011.05.051

[5] Рехвиашвили С.Ш., Алиханов А.А., Алисултанов 3.3. // Поверхность. Рентгеновские, синхронные и нейтронные исследования. 2018. № 4. С. 43-47; Rekhviashvili S.Sh., Alikhanov A.A., Alisultanov Z.Z. // J. Synch. Investig. 2018. V. 12. P. 332-335. doi 10.1134/S1027451018020325

[6] Grassi A., Sironi G., Strini G. // Astrophys. Space Sci. 1986. V. 124. P. 203. doi 10.1007/BF00649761

[7] Landsberg P.T., De Vos A. // J. Phys. A: Math. Gen. 1989. V. 22. P. 1073. doi $10.1088 / 0305-4470 / 22 / 8 / 021$ 
[8] Рехвиашвили С.Ш. // Опт. и спектр. 2020. Т. 128. № 9. C. 1323-1326; Rekhviashvili S.Sh. // Opt. Spectrosc. 2020. V. 128 . P. $1435-1438$. doi 10.1134/S0030400X20090167

[9] Kuzmenko A.B., Van Heumen E., Carbone F., Van der Marel D. // Phys. Rev. Lett. 2008. V. 100. P. 117401. doi 10.1103/PhysRevLett.100.117401

[10] Nair R.R., Blake P., Grigorenko A.N., Novoselov K.S., Booth T.J., Stauber T., Peres N.M.R., Geim A.K. // Science. 2008. V. 320 (5881). P. 1308. doi 10.1126/science. 1156965

[11] Фальковский Л.А. // УФН. 2008. Т. 178. № 9. С. 923-934; Falkovsky L.A. // Phys. Usp. 2008. V. 51. P. 887-897. doi 10.1070/PU2008v051n09ABEH006625

[12] Liu L., Zhou M., Jin L., Li L., Mo Y., Su G., Li X., Zhu H., Tian Y. // Friction. 2019. V. 7. P. 199-216. doi 10.1007/s40544019-0268-4

[13] Dappe Y.J., Basanta M.A., Flores F., Ortega J. // Phys. Rev. B. 2006. V. 74. P. 205434. doi 10.1103/PhysRevB.74.205434

[14] Strek W., Tomala R., Lukaszewicz M., Cichy B., Gerasymchuk Y., Gluchowski P., Marciniak L., Bednarkiewicz A., Hreniak D. // Sci. Rep. 2017. V. 7. P. 41281. doi 10.1038/srep41281

[15] Strek W., Tomala R. // Physica B: Condensed Matter. 2020. V. 579. P. 411840. doi 10.1016/j.physb.2019.411840

[16] Hosmer D.W., Lemeshow S. Applied Logistic Regression. John Wiley \& Sons, 2000. 375 p.

[17] Ландау Л.Д., Лифиии, Е.М. Электродинамика сплошных сред. М.: Наука, 1982. 620 с.

[18] Weber J.W., Bol A.A., Van de Sanden M.C.M. // Appl. Phys. Lett. 2014. V.105. P. 013105. doi 10.1063/1.4889852

[19] Лифшии Е.М., Питаевский Л.П. Физическая кинетика. М.: Наука, 1979. 528 с.

[20] Junaid M., Md Khir M.H., Witjaksono G., Ullah Z., Tansu N., Saheed M.S.M., Kumar P., Hing Wah L., Magsi S.A., Siddiqui M.A. // Molecules. 2020. V. 25. P. 4217. doi $10.3390 /$ molecules 25184217 